Article

\title{
Chemical Composition and in Vitro Antimicrobial and Mutagenic Activities of Seven Lamiaceae Essential Oils
}

\section{Laura De Martino ${ }^{1}$, Vincenzo De Feo ${ }^{1, *}$ and Filomena Nazzaro ${ }^{2}$}

1 Dipartimento di Scienze Farmaceutiche, Università degli Studi di Salerno, via Ponte don Melillo, 84084 Fisciano (Salerno), Italy; E-Mail: 1demartino@unisa.it (L.D.M.)

2 Istituto di Scienze dell'Alimentazione, CNR, via Roma 64, 83100 Avellino, Italy; E-Mail: mena@isa.cnr.it (F.N.)

* Author to whom correspondence should be addressed; E-Mail: defeo@unisa.it; Tel.: +39089969751; Fax: +39089969602.

Received: 6 October 2009; in revised form: 20 October 2009 / Accepted: 20 October 2009 / Published: 20 October 2009

\begin{abstract}
Deeper knowledge of the potentiality of aromatic plants can provide results of economic importance for food and pharmacological industry. The essential oils of seven Lamiaceae species were analyzed by gas chromatography-mass spectrometry and assayed for their antibacterial, antifungal and mutagenic activities. Monoterpenes in the oils ranged between $82.47 \%$ (hyssop oil) and $97.48 \%$ (thyme oil), being mainly represented by oxygenated compounds. The antibacterial activity was evaluated against six pathogenic and five non-pathogenic bacterial strains. Oregano and thyme oils showed the strongest antibacterial activity against the pathogenic ones. The antifungal activity was evaluated against six fungal strains of agrifood interest: the oils tested exhibited variable degrees of activity. Two Salmonella typhimurium strains were used to assess the possible mutagenic activity. No oil showed mutagenic activity. Data obtained let us hypothesise that the use of essential oils could be a viable and safe way to decrease the utilisation of synthetic food preservatives. Further research is needed to obtain information regarding the practical effectiveness of essential oils to prevent the growth of food borne and spoiling microbes under specific application conditions.
\end{abstract}

Keywords: Lamiaceae; essential oils; antimicrobial activity; mutagenic activity 


\section{Introduction}

It is well-known that most spices possess a wide range of biological and pharmacological activities [1]. These volatile compounds are widely used in cosmetics as fragrance components, in the food industry to improve the flavour and the organoleptic properties of different types of foods, and in a variety of household products. In addition to their particular flavour, many essential oils and their isolated components exhibit muscle relaxant, antibacterial and antifungal activities [2]. They are also used as carminative, expectorant, sedative, mucolytic, emmenagogue, spasmolytic, hepatoprotective, antiviral, chemopreventive remedies [3]. Particularly, the antimicrobial and antimutagenic activities of essential oils have formed the basis of many applications, including raw and processed food preservation, pharmaceuticals, and alternative medicine [4]. Restrictions imposed by competent authorities on the use of some synthetic food additives have led to renewed interest in searching for alternatives, like natural antimicrobial compounds [5]. The antimicrobial and preservative activities of spices and essential oils is well documented in many studies. For this reason, these substances have been proposed as safe and effective substitutes of synthetic preservatives [6-9]. The composition of essential oils is variable, depending on the species as well as on the parts of the plant that are utilized [10]. Most of the chemical components of essential oils are terpenoids, including monoterpenes, sesquiterpenes, and their oxygenated derivatives. These low-molecular weight and highly lipophilic compounds easily diffuse across cell membranes to induce biological reactions [11]. Previous studies demonstrated the biological activities of this class of compounds, such as the antibacterial, antifungal, antitermitic, and mosquito larvicidal properties [12]. The genotoxic activity of the essential oil of some Lamiaceae was also evaluated $[1,2,13,14]$. In previous papers we verified the effectiveness of some essential oils against pathogenic and non-pathogenic microbial strains [9]. In addition, we evaluated the antibacterial activity exhibited by some essential oils $[15,16]$ as well some allelopathic properties [17]. Considering the interest of natural products for different commercial fields, a thorough knowledge of the biological and safety profile of essential oils can also give provide results of economic importance [18]. In this light, we studied the composition of the essential oils of seven species of Lamiaceae (Ocimum basilicum L., Hyssopus officinalis L., Lavandula angustifolia Mill., Melissa officinalis L., Origanum vulgare L., Salvia officinalis L., Thymus vulgaris L.), evaluating their antibacterial and antifungal properties against pathogenic or agrifood bacteria or fungal strains. The mutagenic property of the essential oils was also assessed.

\section{Results and Discussion}

\section{Chemical composition of the essential oils}

Table 1 reports the chemical composition of the investigated oils. The main constituents of $M$. officinalis essential oil were (-)-citronellal (39.56\%), carvacrol (13.31\%) and iso-menthone (8.85\%); the main constituents of basil oil were iso-pinocamphone $(35.10 \%)$ and carvone $(39.70 \%)$. The compositions of balm and basil oils agree with literature data [19-22]. $\beta$-Pinene (18.20\%), isopinocamphone $(29.10 \%)$, and trans-pinocamphone (11.12\%) were the most abundant components of H. officinalis essential oil, as reported [23]. Linalool (23.10\%) and linalyl acetate (44.45\%) 
represented the main components of the oil of L. angustifolia; this composition agrees with data reported in literature [24]. The main components of $O$. vulgare and T. vulgaris oils were $o$-cymene and carvacrol, accounting, respectively, for $41.90 \%$ and $44.01 \%$, in oregano, and $56.20 \%$ and $24.44 \%$ in thyme oil. Moreover, the thyme oil also contains a several percentage of thymol $(8.75 \%)$. The oregano oil composition appears to be in part different from others reported in literature: in fact, some papers reported $p$-cymene as the main compound of the oregano oil $[25,26]$. Differences were also reported for the composition of thyme oil [21,27,28]. Sage essential oil was mainly constituted by trans-thujone (37.95\%), camphor (13.92\%) and borneol (7.60\%). This agrees with previous reports [29,30]. All oils tested were mainly constituted by monoterpenes, which represented a percentage ranging between $82.47 \%$, in the hyssop oil, and $97.48 \%$, in the oil of thyme. Among those components, oxygenated monoterpenes were in amounts ranging between $47.46 \%$ (oregano oil) and $87.59 \%$ (lavender oil). Sesquiterpenes were in lower amounts in all the oils analyzed.

\section{Antibacterial activity}

Most of the pathogenic bacteria tested showed an appreciable sensitivity towards the essential oils used in the experiment (Table 2). E. coli and B. cereus 4384 were the most sensitive microorganisms, and, in lesser extent, B. cereus 4313, E. faecalis and $S$. aureus. On the other hand, P. aeruginosa was the most resistant strain, showing a low sensitivity only with the highest amounts (460 and $467 \mu \mathrm{g} /$ paper disc) of thyme and oregano essential oils, respectively. Among the essential oils, oregano and thyme exhibited the most effective antibacterial activity against all the strains tested, in particular against $E$. coli and B. cereus 4384, with inhibition halos of 1.40 and $1.23 \mathrm{~cm}$ for $E$. coli and 1.53 and $1.43 \mathrm{~cm}$ for $B$. cereus 4384, respectively, when the highest doses (467 and $463 \mu \mathrm{g} / \mathrm{paper}$ disc of oregano and thyme essential oils, respectively) were used. Such an activity could be strictly related to their chemical composition: in fact, carvacrol, also found in balm oil, is reported for its good antibacterial effect mainly against $B$. cereus 4384, E. coli and E. faecalis [31].

Activity was also shown by lavender and sage essential oils against the same bacterial strains and with the same amount ( $\sim 50 \mu \mathrm{g} /$ paper disc), with inhibition halos ranging from 0.60 (against $B$. cereus $4313)$ to $0.77 \mathrm{~cm}$ (E. coli). This result could be probably related to the presence of linalool and cisthujone. Basil essential oil was almost totally ineffective, exhibiting a weak antimicrobial action only against $E$. coli ( $0.60 \mathrm{~cm}$ inhibition halo), with the highest dose used (480 $\mu \mathrm{g} /$ paper disc).

The effectiveness demonstrated by all the essential oils against the pathogenic microorganism $E$. coli DSM 8579 serotype O157:H7, a pathogen of worldwide human health interest, can be considered important, as this strain produces verocytotoxins, and causes haemorrhagic colitis and haemolytic uremic syndrome. Moreover, the activity against both B. cereus strains could led to the use of the tested oils, except basil, as natural food preservatives against this strain, strictly linked to food-borne illnesses [32]. All Lactobacilli tested did not show any inhibition of such useful bacteria. Moreover, the available literature reports that some spices can positively influence the technological and biological performances of lactic acid bacteria, such as $L b$. sakei, involved as starter in the manufacturing of sausages and they can improve the shelf life and safety of the final product [33]. Due to the overall activities demonstrated by oregano and thyme oils, and, in less extent, by hyssop and balm oils, their use could be useful in food technology. 
Table 1. Chemical composition of essential oils: Melissa officinalis L. (Balm), Ocimum basilicum L. (Basil), Hyssopus officinalis L. (Hyssop), Lavandula angustifolia Mill. (Lavender), Origanum vulgare L. (Oregano), Salvia officinalis L. (Sage), Thymus vulgaris L. (Thyme).

\begin{tabular}{|c|c|c|c|c|c|c|c|c|c|c|}
\hline Compound & $\mathbf{K i}^{\mathbf{a}}$ & $\mathbf{K i}^{\mathbf{b}}$ & Balm & Basil & Hyssop & Lavender & Oregano & Sage & Thyme & Identification $^{\mathrm{d}}$ \\
\hline & & & $\%^{\mathrm{c}}$ & $\%$ & $\%$ & $\%$ & $\%$ & $\%$ & $\%$ & \\
\hline$\alpha$-Thujene & 930 & 1035 & $0.12 \pm 0.01$ & $\mathrm{~T}$ & $0.41 \pm 0.02$ & $0.23 \pm 0.03$ & $0.46 \pm 0.01$ & $0.45 \pm 0.01$ & $\mathrm{~T}$ & LRI, MS \\
\hline$\alpha$-Pinene & 938 & 1032 & $0.90 \pm 0.02$ & $0.3 \pm 0.01$ & $1.05 \pm 0.04$ & --- & $0.45 \pm 0.03$ & $4.44 \pm 0.12$ & $2.54 \pm 0.15$ & LRI, MS, Co-GC \\
\hline (-)-Camphene & 953 & 1076 & --- & --- & $0.25 \pm 0.04$ & $0.67 \pm 0.05$ & $0.17 \pm 0.04$ & $4.09 \pm 0.05$ & $0.96 \pm 0.07$ & LRI, MS, Co-GC \\
\hline Sabinene & 973 & 1132 & $\mathrm{~T}$ & $0.27 \pm 0.05$ & $1.43 \pm 0.90$ & $\mathrm{~T}$ & $\mathrm{~T}$ & $0.44 \pm 0.01$ & $\mathrm{~T}$ & RI, MS, Co-GC \\
\hline Hepten-3-one & 975 & & $\mathrm{~T}$ & --- & --- & --- & --- & --- & --- & LRI, MS \\
\hline$\beta$-Pinene & 978 & 1118 & $0.43 \pm 0.06$ & $0.46 \pm 0.03$ & $18.20 \pm 0.05$ & --- & $0.21 \pm 0.05$ & $2.54 \pm 0.07$ & --- & LRI, MS, Co-GC \\
\hline cis-Pinane & 980 & & --- & $0.10 \pm 0.03$ & --- & $0.11 \pm 0.02$ & $0.14 \pm 0.05$ & --- & --- & LRI, MS \\
\hline Verbenene & 982 & & $\mathrm{~T}$ & $\mathrm{~T}$ & 0.1 & $\mathrm{~T}$ & $\mathrm{~T}$ & $\mathrm{~T}$ & $\mathrm{~T}$ & LRI, MS \\
\hline Myrcene & 993 & 1174 & $0.10 \pm 0.03$ & $0.34 \pm 0.06$ & $1.84 \pm 0.20$ & $0.34 \pm 0.03$ & $0.50 \pm 0.03$ & $0.51 \pm 0.06$ & $0.10 \pm 0.02$ & LRI, MS, Co-GC \\
\hline$\alpha$-Phellandrene & 995 & 1176 & $\mathrm{~T}$ & $\mathrm{~T}$ & $\mathrm{~T}$ & $0.20 \pm 0.03$ & $0.15 \pm 0.01$ & $\mathrm{~T}$ & $\mathrm{~T}$ & LRI, MS, Co-GC \\
\hline$\Delta 3$-Carene & 997 & 1153 & --- & --- & --- & $0.27 \pm 0.06$ & $0.18 \pm 0.02$ & --- & --- & LRI, MS, Co-GC \\
\hline$\alpha$-Terpinene & 1012 & 1188 & $0.15 \pm 0.08$ & $\mathrm{~T}$ & $0.18 \pm 0.06$ & $\mathrm{~T}$ & $0.46 \pm 0.05$ & $\mathrm{~T}$ & $0.11 \pm 0.01$ & LRI, MS, Co-GC \\
\hline$o$-Cymene & 1020 & 1187 & $2.33 \pm 0.91$ & $0.15 \pm 0.02$ & $0.23 \pm 0.02$ & $0.65 \pm 0.08$ & $41.90 \pm 0.10$ & $2.53 \pm 0.20$ & $56.20 \pm 0.20$ & LRI, MS, Co-GC \\
\hline p-Cymene & 1024 & 1280 & $0.60 \pm 0.01$ & --- & --- & $0.34 \pm 0.05$ & $0.12 \pm 0.02$ & $1.25 \pm 0.11$ & $0.15 \pm 0.05$ & LRI, MS, Co-GC \\
\hline$\beta$-Phellandrene & 1029 & 1218 & $0.33 \pm 0.01$ & $0.32 \pm 0.02$ & $1.85 \pm 0.20$ & $0.12 \pm 0.01$ & $0.14 \pm 0.03$ & $0.97 \pm 0.04$ & $0.16 \pm 0.08$ & LRI, MS, Co-GC \\
\hline Limonene & 1030 & 1203 & $1.44 \pm 0.30$ & $0.38 \pm 0.01$ & $1.27 \pm 0.68$ & $0.27 \pm 0.02$ & $0.35 \pm 0.03$ & $1.36 \pm 0.04$ & $0.64 \pm 0.01$ & LRI, MS, Co-GC \\
\hline 1,8-Cineole & 1034 & 1213 & $0.22 \pm 0.00$ & $0.55 \pm 0.09$ & $0.17 \pm 0.02$ & $\mathrm{~T}$ & $0.61 \pm 0.06$ & $4.20 \pm 0.30$ & $\mathrm{~T}$ & LRI, MS \\
\hline (Z)- $\beta$-Ocimene & 1038 & 1246 & $\mathrm{~T}$ & $0.10 \pm 0.01$ & $0.33 \pm 0.04$ & $1.66 \pm 0.30$ & $\mathrm{~T}$ & $\mathrm{~T}$ & $\mathrm{~T}$ & LRI, MS, Co-GC \\
\hline (E)- $\beta$-Ocimene & 1049 & 1280 & $\mathrm{~T}$ & $1.21 \pm 0.04$ & $0.98 \pm 0.05$ & $0.60 \pm 0.06$ & $\mathrm{~T}$ & $\mathrm{~T}$ & $\mathrm{~T}$ & LRI, MS, Co-GC \\
\hline$\gamma$-Terpinene & 1057 & 1255 & $0.36 \pm 0.00$ & $\mathrm{~T}$ & $0.18 \pm 0.02$ & $\mathrm{~T}$ & $2.84 \pm 0.20$ & $0.10 \pm 0.00$ & $0.44 \pm 0.01$ & LRI, MS, Co-GC \\
\hline cis-Sabinene hydrate & 1063 & 1556 & --- & --- & --- & $0.33 \pm 0.02$ & $0.17 \pm 0.03$ & $0.15 \pm 0.01$ & --- & LRI, MS, Co-GC \\
\hline cis-Linalool oxide & 1065 & 1450 & --- & --- & --- & $0.45 \pm 0.06$ & --- & --- & --- & LRI, MS, Co-GC \\
\hline Fenchone & 1067 & 1392 & --- & $0.44 \pm 0.08$ & --- & --- & --- & --- & --- & LRI, MS \\
\hline Terpinolene & 1086 & 1265 & $0.13 \pm 0.04$ & $0.12 \pm 0.06$ & $0.18 \pm 0.04$ & $\mathrm{~T}$ & $0.11 \pm 0.01$ & $\mathrm{~T}$ & $0.66 \pm 0.07$ & LRI, MS \\
\hline
\end{tabular}


Table 1. Cont.

\begin{tabular}{|c|c|c|c|c|c|c|c|c|c|c|}
\hline Linalool & 1097 & 1553 & $0.68 \pm 0.06$ & $0.70 \pm 0.01$ & $1.03 \pm 0.10$ & $23.10 \pm 0.20$ & $0.72 \pm 0.30$ & $1.10 \pm 0.60$ & $0.39 \pm 0.07$ & LRI, MS, Co-GC \\
\hline endo-Fenchol & 1098 & & --- & $0.24 \pm 0.01$ & --- & --- & --- & --- & --- & LRI, MS \\
\hline cis-Thujone & 1105 & 1430 & $\mathrm{~T}$ & --- & $0.10 \pm 0.01$ & $\mathrm{~T}$ & $\mathrm{~T}$ & --- & $\mathrm{T}$ & LRI, MS, Co-GC \\
\hline trans-Thujone & 1115 & 1449 & --- & --- & --- & --- & --- & $37.95 \pm 0.80$ & --- & LRI, MS, Co-GC \\
\hline trans-Pinocarveol & 1138 & 1654 & --- & $\mathrm{T}$ & $0.14 \pm 0.01$ & $\mathrm{~T}$ & $\mathrm{~T}$ & $0.18 \pm 0.03$ & $\mathrm{~T}$ & LRI, MS \\
\hline (-)-Citronellal & 1143 & 1491 & $39.56 \pm 0.40$ & --- & --- & -- & --- & $0.22 \pm 0.01$ & $0.54 \pm 0.08$ & LRI, MS, Co-GC \\
\hline iso-Borneol & 1144 & 1633 & $0.55 \pm 0.01$ & --- & --- & --- & --- & --- & $0.12 \pm 0.02$ & LRI, MS, Co-GC \\
\hline Camphor & 1145 & 1532 & $1.10 \pm 0.01$ & $0.66 \pm 0.02$ & --- & $0.94 \pm 0.03$ & $\mathrm{~T}$ & $13.92 \pm 0.70$ & $\mathrm{~T}$ & LRI, MS, Co-GC \\
\hline Menthofuran & 1150 & 1502 & --- & --- & $0.33 \pm 0.01$ & --- & --- & --- & --- & LRI, MS, Co-GC \\
\hline iso-Pinocamphone & 1153 & 1566 & $\mathrm{~T}$ & $35.10 \pm 0.02$ & $29.10 \pm 0.02$ & $0.10 \pm 0.02$ & $0.10 \pm 0.02$ & $0.10 \pm 0.02$ & $\mathrm{~T}$ & LRI, MS \\
\hline trans-Pinocamphone & 1159 & & $0.44 \pm 0.02$ & $\mathrm{~T}$ & $11.12 \pm 0.90$ & --- & $\mathrm{T}$ & $0.27 \pm 0.02$ & $\mathrm{~T}$ & LRI, MS \\
\hline Lavandulol & 1162 & 1674 & --- & --- & $4.43 \pm 0.40$ & --- & --- & --- & --- & LRI, MS \\
\hline iso-Menthone & 1163 & 1503 & $8.85 \pm 0.90$ & --- & --- & --- & --- & --- & $0.11 \pm 0.02$ & LRI, MS, Co-GC \\
\hline Pinocarvone & 1165 & 1587 & $\mathrm{~T}$ & $0.43 \pm 0.01$ & $0.46 \pm 0.04$ & $\mathrm{~T}$ & $\mathrm{~T}$ & $\mathrm{~T}$ & $\mathrm{~T}$ & LRI, MS \\
\hline Borneol & 1167 & 1719 & $0.15 \pm 0.01$ & $0.21 \pm 0.02$ & $0.14 \pm 0.03$ & $6.35 \pm 0.94$ & $0.26 \pm 0.02$ & $7.60 \pm 0.40$ & $0.17 \pm 0.05$ & LRI, MS, Co-GC \\
\hline Terpinen-4-ol & 1176 & 1611 & $0.12 \pm 0.02$ & $0.22 \pm 0.01$ & $0.26 \pm 0.06$ & $0.25 \pm 0.03$ & $0.45 \pm 0.03$ & $0.55 \pm 0.02$ & $\mathrm{~T}$ & LRI, MS, Co-GC \\
\hline dihydro-Carveol & 1177 & & --- & --- & $1.25 \pm 0.15$ & $0.36 \pm 0.03$ & --- & $0.20 \pm 0.01$ & $0.22 \pm 0.01$ & LRI, MS \\
\hline$p$-Cymen-8-ol & 1185 & 1864 & $\mathrm{~T}$ & --- & $\mathrm{T}$ & $0.28 \pm 0.01$ & $0.18 \pm 0.05$ & $0.11 \pm 0.02$ & $\mathrm{~T}$ & LRI, MS \\
\hline$\alpha$-Terpineol & 1189 & 1706 & $0.11 \pm 0.01$ & $1.30 \pm 0.30$ & $1.24 \pm 0.10$ & $0.38 \pm 0.05$ & $\mathrm{~T}$ & $0.27 \pm 0.01$ & $0.26 \pm 0.04$ & LRI, MS, Co-GC \\
\hline Myrtenal & 1193 & 1648 & --- & $1.01 \pm 0.03$ & $1.05 \pm 0.30$ & $0.37 \pm 0.06$ & --- & $0.22 \pm 0.04$ & $0.26 \pm 0.05$ & LRI, MS \\
\hline Estragole & 1195 & 1670 & --- & --- & $0.44 \pm 0.03$ & --- & $0.11 \pm 0.01$ & $\mathrm{~T}$ & --- & LRI, MS, Co-GC \\
\hline Myrtenol & 1196 & 1804 & --- & $0.61 \pm 0.02$ & $1.26 \pm 0.50$ & $0.37 \pm 0.03$ & --- & $0.25 \pm 0.01$ & $0.26 \pm 0.04$ & LRI, MS \\
\hline Citronellol & 1213 & 1772 & $6.20 \pm 0.29$ & --- & --- & --- & --- & --- & --- & LRI, MS, Co-GC \\
\hline cis-Carveol & 1226 & 1878 & --- & $0.11 \pm 0.01$ & --- & --- & --- & --- & --- & LRI, MS \\
\hline Carvone & 1241 & 1752 & --- & $39.70 \pm 0.90$ & --- & --- & --- & --- & --- & LRI, MS, Co-GC \\
\hline Linalyl acetate & 1248 & 1565 & $2.30 \pm 0.26$ & $0.44 \pm 0.02$ & $0.33 \pm 0.01$ & $44.45 \pm 0.70$ & $0.10 \pm 0.01$ & $1.55 \pm 0.20$ & --- & LRI, MS, Co-GC \\
\hline Geraniol & 1255 & 1857 & $5.70 \pm 0.30$ & --- & --- & $9.26 \pm 0.26$ & --- & $0.33 \pm 0.02$ & --- & LRI, MS \\
\hline cis-Anethole & 1262 & & --- & --- & $0.35 \pm 0.05$ & --- & --- & --- & --- & LRI, MS \\
\hline Isobornyl acetate & 1277 & & $\mathrm{~T}$ & $\mathrm{~T}$ & --- & $0.26 \pm 0.01$ & $\mathrm{~T}$ & $0.68 \pm 0.02$ & $\mathrm{~T}$ & LRI, MS \\
\hline
\end{tabular}


Table 1. Cont

\begin{tabular}{|c|c|c|c|c|c|c|c|c|c|c|}
\hline Bornyl acetate & 1284 & 1591 & $\mathrm{~T}$ & $\mathrm{~T}$ & $\mathrm{~T}$ & $0.24 \pm 0.03$ & $\mathrm{~T}$ & $0.88 \pm 0.04$ & $\mathrm{~T}$ & LRI, MS \\
\hline $\begin{array}{l}\text { Cinnamic } \\
\text { methyl ester }\end{array}$ & 1289 & & --- & $0.11 \pm 0.03$ & --- & --- & --- & --- & --- & LRI, MS \\
\hline Thymol & 1290 & 2198 & $0.11 \pm 0.03$ & --- & $\mathrm{T}$ & --- & $0.75 \pm 0.02$ & $\mathrm{~T}$ & $8.75 \pm 0.90$ & LRI, MS, Co-GC \\
\hline Carvacrol & 1297 & 2239 & $13.31 \pm 0.90$ & $\mathrm{~T}$ & $\mathrm{~T}$ & --- & $44.01 \pm 0.90$ & $0.26 \pm 0.01$ & $24.44 \pm 0.90$ & LRI, MS, Co-GC \\
\hline Myrtenyl acetate & 1313 & & --- & $0.51 \pm 0.03$ & $0.56 \pm 0.02$ & --- & $\mathrm{T}$ & $\mathrm{T}$ & --- & LRI, MS \\
\hline Eugenol & 1353 & 2186 & $0.55 \pm 0.03$ & --- & --- & --- & --- & --- & --- & LRI, MS, Co-GC \\
\hline Citronellyl acetate & 1358 & 1662 & $1.60 \pm 0.90$ & --- & --- & --- & --- & --- & --- & LRI, MS \\
\hline Methyl eugenol & 1369 & 2023 & $\mathrm{~T}$ & $0.53 \pm 0.03$ & $0.67 \pm 0.01$ & $\mathrm{~T}$ & $\mathrm{~T}$ & --- & --- & LRI, MS \\
\hline$\alpha$-Copaene & 1377 & 1497 & $\mathrm{~T}$ & $0.11 \pm 0.01$ & $0.15 \pm 0.02$ & $\mathrm{~T}$ & $0.15 \pm 0.04$ & $\mathrm{~T}$ & $\mathrm{~T}$ & LRI, MS \\
\hline Geranyl acetate & 1379 & 1765 & $1.70 \pm 0.30$ & --- & --- & --- & --- & --- & --- & LRI, MS \\
\hline Isoledene & 1382 & & $\mathrm{~T}$ & $0.10 \pm 0.01$ & $0.11 \pm 0.01$ & $\mathrm{~T}$ & $0.11 \pm 0.02$ & $\mathrm{~T}$ & $\mathrm{~T}$ & LRI, MS \\
\hline$\beta$-Bourbonene & 1385 & 1535 & --- & $1.20 \pm 0.32$ & $1.34 \pm 0.30$ & --- & --- & --- & --- & LRI, MS \\
\hline$\beta$-Elemene & 1387 & 1600 & $0.60 \pm 0.01$ & $0.11 \pm 0.01$ & $\mathrm{~T}$ & --- & $\mathrm{T}$ & --- & $\mathrm{T}$ & LRI, MS \\
\hline$\alpha$-Gurjunene & 1408 & 1529 & $0.40 \pm 0.01$ & $0.41 \pm 0.04$ & $0.55 \pm 0.04$ & --- & --- & --- & --- & LRI, MS \\
\hline Longifolene & 1411 & 1576 & $0.90 \pm 0.12$ & $0.52 \pm 0.01$ & $0.53 \pm 0.02$ & $\mathrm{~T}$ & $\mathrm{~T}$ & $\mathrm{~T}$ & $\mathrm{~T}$ & LRI, MS \\
\hline$\beta$-Caryophyllene & 1418 & 1612 & $0.62 \pm 0.01$ & $1.40 \pm 0.50$ & $1.00 \pm 0.50$ & $1.05 \pm 0.90$ & $0.22 \pm 0.09$ & $1.28 \pm 0.03$ & $0.15 \pm 0.01$ & LRI, MS \\
\hline$\beta$-Cedrene & 1424 & 1638 & $0.34 \pm 0.04$ & $0.55 \pm 0.02$ & $0.56 \pm 0.01$ & $1.35 \pm 0.15$ & $0.56 \pm 0.03$ & $1.00 \pm 0.01$ & --- & LRI, MS \\
\hline Aromadendrene & 1437 & 1628 & $\mathrm{~T}$ & $\mathrm{~T}$ & $\mathrm{~T}$ & $\mathrm{~T}$ & $\mathrm{~T}$ & 0.1 & $\mathrm{~T}$ & LRI, MS \\
\hline$\alpha$-Humulene & 1455 & 1689 & $0.22 \pm 0.01$ & $0.55 \pm 0.04$ & $0.57 \pm 0.03$ & $0.56 \pm 0.04$ & $0.11 \pm 0.02$ & $5.90 \pm 0.90$ & $\mathrm{~T}$ & LRI, MS \\
\hline allo-Aromadendrene & 1463 & 1661 & $\mathrm{~T}$ & $1.23 \pm 0.50$ & $1.36 \pm 0.20$ & $0.47 \pm 0.04$ & $\mathrm{~T}$ & $0.11 \pm 0.02$ & $\mathrm{~T}$ & LRI, MS \\
\hline Neryl isobutyrate & 1468 & & --- & --- & --- & $0.10 \pm 0.01$ & --- & --- & --- & LRI, MS \\
\hline$\gamma$-Gurjunene & 1473 & 1687 & $0.22 \pm 0.01$ & $0.51 \pm 0.02$ & $0.10 \pm 0.01$ & --- & $0.11 \pm 0.01$ & $0.13 \pm 0.05$ & $\mathrm{~T}$ & LRI, MS \\
\hline cis- $\beta$-Guaiene & 1490 & 1694 & $0.15 \pm 0.02$ & --- & $0.44 \pm 0.01$ & --- & --- & --- & --- & LRI, MS \\
\hline Bicyclogermacrene & 1491 & 1756 & --- & $1.50 \pm 0.02$ & $3.10 \pm 0.50$ & --- & --- & --- & --- & LRI, MS \\
\hline $\begin{array}{l}\text { cis-Muurola-4(14),5- } \\
\text { diene }\end{array}$ & 1510 & 1675 & $2.30 \pm 0.50$ & $3.01 \pm 0.90$ & $3.68 \pm 0.90$ & $0.29 \pm 0.02$ & $\mathrm{~T}$ & $\mathrm{~T}$ & $\mathrm{~T}$ & LRI, MS \\
\hline$\alpha$-7-epi-Selinene & 1518 & 1740 & $0.64 \pm 0.02$ & $0.11 \pm 0.01$ & $0.15 \pm 0.02$ & --- & $0.14 \pm 0.03$ & $0.11 \pm 0.01$ & $\mathrm{~T}$ & LRI, MS \\
\hline Caryophyllene oxide & 1580 & 2008 & $0.22 \pm 0.02$ & --- & --- & $0.44 \pm 0.02$ & $0.18 \pm 0.02$ & $0.77 \pm 0.05$ & --- & LRI, MS \\
\hline
\end{tabular}


Table 1. Cont.

\begin{tabular}{|c|c|c|c|c|c|c|c|c|c|}
\hline$\alpha$-Cadinol & 2255 & $0.20 \pm 0.01$ & --- & $0.35 \pm 0.02$ & --- & --- & --- & --- & LRI, MS \\
\hline Total compounds & & 96.95 & 97.93 & 96.81 & 97.21 & 97.22 & 97.98 & 97.63 & \\
\hline Monoterpenes hydrocarbons & & 6.89 & 3.75 & 28.39 & 5.46 & 48.18 & 18.68 & 61.96 & \\
\hline Oxigenated monoterpenes & & 83.25 & 82.76 & 54.08 & 87.59 & 47.46 & 70.99 & 35.52 & \\
\hline Total Monoterpenes & & 90.14 & 86.51 & 82.47 & 93.05 & 95.64 & 89.67 & 97.48 & \\
\hline Sesquiterpenes hydrocarbons & & 6.39 & 11.31 & 13.64 & 3.72 & 1.4 & 7.54 & 0.15 & \\
\hline Oxigenated sesquiterpenes & & 0.42 & 0 & 0.35 & 0.44 & 0.18 & 0.77 & 0 & \\
\hline Total Sesquiterpenes & & 6.81 & 11.31 & 13.99 & 4.16 & 1.58 & 8.31 & 0.15 & \\
\hline Non terpenes & & 0 & 0.22 & 0.35 & 0 & 0 & 0 & 0 & \\
\hline
\end{tabular}

The values are the mean of three replicates $\pm \mathrm{SD} .{ }^{\mathrm{a}}: \mathrm{HP} 5 \mathrm{MS}$ column; ${ }^{\mathrm{b}}: \mathrm{HP}$ Innowax column $;{ }^{\mathrm{c}}: \mathrm{LRI}=$ linear retention index, MS $=$ mass spectrum, Co-GC $=$ co-injection with authentic compound; ${ }^{\mathrm{d}}$ : Mass of compounds in $\mathrm{mg} / 100 \mathrm{mg}$ oil; $\mathrm{t}$, trace $(<0.05 \%)$; mean value \pm standard error, $n$, three independent determinations.

Table 2. Inhibition of bacterial growth of essential oils: Melissa officinalis L. (balm), Ocimum basilicum L. (basil), Hyssopus officinalis L. (hyssop), Lavandula angustifolia Mill. (lavender), Origanum vulgare L. (oregano), Salvia officinalis L. (sage), Thymus vulgaris L. (thyme). Data are expressed in $\mathrm{cm}$ and do not include the diameter of paper disc. Results are shown as mean \pm standard deviation (SD) of the inhibition zone $(\mathrm{n}=3)$.

\begin{tabular}{lcccccc}
$\begin{array}{l}\text { Essential oil } \\
(\boldsymbol{\mu g} / \text { paper disc) }\end{array}$ & $\begin{array}{c}\text { Bacillus } \\
\text { cereus 4313 }\end{array}$ & $\begin{array}{c}\text { Bacillus } \\
\text { cereus } \mathbf{4 3 8 4}\end{array}$ & $\begin{array}{c}\text { Pseudomonas } \\
\text { aeruginosa }\end{array}$ & $\begin{array}{c}\text { Escherichia } \\
\text { coli }\end{array}$ & $\begin{array}{c}\text { Enterococcus } \\
\text { faecalis }\end{array}$ & $\begin{array}{c}\text { Staphylococcus } \\
\text { aureus }\end{array}$ \\
\cline { 2 - 6 } & $\mathrm{IZ}( \pm \mathrm{SD})$ & $\mathrm{IZ}( \pm \mathrm{SD})$ & $\mathrm{IZ}( \pm \mathrm{SD})$ & $\mathrm{IZ}( \pm \mathrm{SD})$ & $\mathrm{IZ}( \pm \mathrm{SD})$ & $\mathrm{IZ}( \pm \mathrm{SD})$ \\
\hline Balm $\mathbf{8 8} \boldsymbol{\mu g}$ & $\mathrm{NA}( \pm 0.00)$ & $\mathrm{NA}( \pm 0.00)$ & $\mathrm{NA}( \pm 0.00)$ & $\mathrm{NA}( \pm 0.00)$ & $\mathrm{NA}( \pm 0.00)$ & $\mathrm{NA}( \pm 0.00)$ \\
Balm 177 $\boldsymbol{\mu g}$ & $\mathrm{NA}( \pm 0.00)$ & $0.75( \pm 0.07)$ & $\mathrm{NA}( \pm 0.00)$ & $0.65( \pm 0.07)$ & $0.80( \pm 0.20)$ & $\mathrm{NA}( \pm 0.00)$ \\
Balm 442 $\boldsymbol{\mu g}$ & $0.63( \pm 0.05)$ & $1.00( \pm 0.00)$ & $\mathrm{NA}( \pm 0.00)$ & $0.67( \pm 0.06)$ & $1.30( \pm 0.14)$ & $0.60( \pm 0.05)$ \\
& & & & & \\
Basil 96 $\boldsymbol{\mu g}$ & $\mathrm{NA}( \pm 0.00)$ & $\mathrm{NA}( \pm 0.00)$ & $\mathrm{NA}( \pm 0.00)$ & $\mathrm{NA}( \pm 0.00)$ & $\mathrm{NA}( \pm 0.00)$ & $\mathrm{NA}( \pm 0.00)$ \\
Basil 192 $\boldsymbol{\mu g}$ & $\mathrm{NA}( \pm 0.00)$ & $\mathrm{NA}( \pm 0.00)$ & $\mathrm{NA}( \pm 0.00)$ & $\mathrm{NA}( \pm 0.00)$ & $\mathrm{NA}( \pm 0.00)$ & $\mathrm{NA}( \pm 0.00)$ \\
\hline
\end{tabular}


Table 2. Cont

\begin{tabular}{|c|c|c|c|c|c|c|}
\hline Basil $480 \mu \mathrm{g}$ & $\mathrm{NA}( \pm 0.00)$ & $\mathrm{NA}( \pm 0.00)$ & $\mathrm{NA}( \pm 0.00)$ & $0.60( \pm 0.04)$ & $\mathrm{NA}( \pm 0.00)$ & $\mathrm{NA}( \pm 0.00)$ \\
\hline Hyssop 93 нg & $\mathrm{NA}( \pm 0.00)$ & $\mathrm{NA}( \pm 0.00)$ & $\mathrm{NA}( \pm 0.00)$ & $\mathrm{NA}( \pm 0.00)$ & $\mathrm{NA}( \pm 0.00)$ & $\mathrm{NA}( \pm 0.00)$ \\
\hline Hyssop $185 \mu \mathrm{g}$ & $\mathrm{NA}( \pm 0.00)$ & $\mathrm{NA}( \pm 0.00)$ & $\mathrm{NA}( \pm 0.00)$ & $\mathrm{NA}( \pm 0.00)$ & $\mathrm{NA}( \pm 0.00)$ & $\mathrm{NA}( \pm 0.00)$ \\
\hline Hyssop $463 \mu \mathrm{g}$ & $0.63( \pm 0.06)$ & $0.60( \pm 0.05)$ & $\mathrm{NA}( \pm 0.00)$ & $0.77( \pm 0.12)$ & $0.60( \pm 0.04)$ & $0.60( \pm 0.00)$ \\
\hline Lavender $87 \mu \mathrm{g}$ & $\mathrm{NA}( \pm 0.00)$ & $\mathrm{NA}( \pm 0.00)$ & $\mathrm{NA}( \pm 0.00)$ & $\mathrm{NA}( \pm 0.00)$ & $\mathrm{NA}( \pm 0.00)$ & $\mathrm{NA}( \pm 0.00)$ \\
\hline Lavender $177 \mu \mathrm{g}$ & $\mathrm{NA}( \pm 0.00)$ & $\mathrm{NA}( \pm 0.00)$ & $\mathrm{NA}( \pm 0.00)$ & $\mathrm{NA}( \pm 0.00)$ & $\mathrm{NA}( \pm 0.00)$ & $\mathrm{NA}( \pm 0.00)$ \\
\hline Lavender $443 \mu \mathrm{g}$ & $0.63( \pm 0.06)$ & $0.73( \pm 0.15)$ & $\mathrm{NA}( \pm 0.00)$ & $0.73( \pm 0.06)$ & $\mathrm{NA}( \pm 0.00)$ & $0.75( \pm 0.17)$ \\
\hline Oregano $93 \mu \mathrm{g}$ & $0.70( \pm 0.14)$ & $0.9( \pm 0.14)$ & $\mathrm{NA}( \pm 0.00)$ & $0.77( \pm 0.11)$ & $\mathrm{NA}( \pm 0.00)$ & $\mathrm{NA}( \pm 0.00)$ \\
\hline Oregano $187 \mu \mathrm{g}$ & $0.80( \pm 0.20)$ & $1.00( \pm 0.0)$ & $\mathrm{NA}( \pm 0.00)$ & $0.97( \pm 0.06)$ & $0.83( \pm 0.06)$ & $0.65( \pm 0.08)$ \\
\hline Oregano $467 \mu \mathrm{g}$ & $1.10( \pm 0.26)$ & $1.53( \pm 0.06)$ & $0.90( \pm 0.17)$ & $1.40( \pm 0.20)$ & $1.07( \pm 0.32)$ & $0.70( \pm 0.00)$ \\
\hline Sage $91 \mu \mathrm{g}$ & $\mathrm{NA}( \pm 0.00)$ & $\mathrm{NA}( \pm 0.00)$ & $\mathrm{NA}( \pm 0.00)$ & $\mathrm{NA}( \pm 0.00)$ & $\mathrm{NA}( \pm 0.00)$ & $\mathrm{NA}( \pm 0.00)$ \\
\hline Sage $183 \mu \mathrm{g}$ & $\mathrm{NA}( \pm 0.00)$ & $\mathrm{NA}( \pm 0.00)$ & $\mathrm{NA}( \pm 0.00)$ & $\mathrm{NA}( \pm 0.00)$ & $\mathrm{NA}( \pm 0.00)$ & $\mathrm{NA}( \pm 0.00)$ \\
\hline Sage $457 \mu \mathrm{g}$ & $0.60( \pm 0.04)$ & $0.63( \pm 0.06)$ & $\mathrm{NA}( \pm 0.00)$ & $0.77( \pm 0.06)$ & $\mathrm{NA}( \pm 0.00)$ & $0.70( \pm 0.40)$ \\
\hline Thyme $93 \mu \mathrm{g}$ & $\mathrm{NA}( \pm 0.00)$ & $0.80( \pm 0.20)$ & $\mathrm{NA}( \pm 0.00)$ & $0.73( \pm 0.06)$ & $0.70( \pm 0.1)$ & $\mathrm{NA}( \pm 0.00)$ \\
\hline Thyme $185 \mu \mathrm{g}$ & $\mathrm{NA}( \pm 0.00)$ & $0.87( \pm 0.15)$ & $\mathrm{NA}( \pm 0.00)$ & $0.87( \pm 0.06)$ & $0.73( \pm 0.06)$ & $0.70( \pm 0.12)$ \\
\hline Thyme $463 \mu \mathrm{g}$ & $0.75( \pm 0.11)$ & $1.43( \pm 0.06)$ & $0.70( \pm 0.00)$ & $1.23( \pm 0.06)$ & $1.07( \pm 0.40)$ & $0.80( \pm 0.0)$ \\
\hline Gentamycin $8 \mu \mathrm{g}$ & $1.77( \pm 0.12)$ & $1.57( \pm 0.12)$ & $1.53( \pm 0,06)$ & $1.57( \pm 0.12)$ & $1.97( \pm 0.06)$ & $0.57( \pm 0.12)$ \\
\hline Chloramphenicol & $1.13( \pm 0.06)$ & $1.37( \pm 0.06)$ & $0.67( \pm 0.06)$ & $1.03( \pm 0.06)$ & $2.13( \pm 0.12)$ & $0.83( \pm 0.29)$ \\
\hline Tetracicline $7 \mu \mathrm{g}$ & $1.03( \pm 0.06)$ & $0.83( \pm 0.06)$ & $0.97( \pm 0.06)$ & $1.27( \pm 0.12)$ & $1.37( \pm 0.12)$ & $0.57( \pm 0.06)$ \\
\hline DMSO & $\mathrm{NA}( \pm 0.00)$ & $\mathrm{NA}( \pm 0.00)$ & $\mathrm{NA}( \pm 0.00)$ & $\mathrm{NA}( \pm 0.00)$ & $\mathrm{NA}( \pm 0.00)$ & $\mathrm{NA}( \pm 0.00)$ \\
\hline
\end{tabular}

$\mathrm{NA}=$ not active. Lactobacillus acidophilus, Lactobacillus casei, Lactobacillus bulgaricus, Lactobacillus sakei, Lactobacillus rhamnosus. 


\section{Antifungal activity}

We tested the action of essential oils against different species of the genus Penicillium, an important contaminant of foods and agricultural commodities, against one strain of A. pullulans, usually recognised as contaminant of vegetables [34] and against D. hansenii, an ubiquitous unicellular haploid yeast, commonly found in freshwater and seawater or as opportunistic parasite, in humans, fish and vegetables [35].

The results in Table 3, show that the essential oils exhibited variable degrees of antifungal activity. Generally, all oils exhibited good antifungal capabilities, in particular against $A$. pullulans and $P$. simplicissimum (at the three doses used, ranging from 87 to $480 \mu \mathrm{g} / \mathrm{paper}$ disc), in a dose-dependent manner. A good activity against $P$. citrinum was also recorded. Fungal growth was reduced by all essential oils, with inhibition halos ranging from 0.20 (oregano oil), to $1.43 \mathrm{~cm}$ (balm). This result appears of importance, being this microorganism a well known producer of the toxic metabolite citrinin, a hepatonephrotoxic mycotoxin involved in different disease outbreaks in animals and humans [36].

No activity was recorded against $D$. hansenii, except when using the highest dose of oregano and sage essential oil (inhibition halos 0.83 and $0.23 \mathrm{~cm}$, respectively) and against $P$. expansum (by using $467 \mu \mathrm{g}$ of oregano essential oil). A weak activity was detected toward P. aurantiogriseum, only for balm, hyssop and lavender essential oils at different doses.

Our data agree with available literature. Antifungal activity of several essential oils has been previously reported [37]. T. vulgaris oil was found to exhibit an inhibitory activity against Aspergillus flavus [38]. The antifungal activity of $H$. officinalis oil was previously tested against phytopathogenic fungi, Pyrenophora avenae and Pyricularia oryzae [39]. S. officinalis oil, like other essential oils, presented a broad antifungal spectrum [40,41]. The available literature reports also the antifungal activity showed by essential oils and some of their main constituents $[42,43]$.

\section{Mutagenic activity}

None of the essential oils tested, at any dose, showed mutagenic activity on TA98 and TA100 Salmonella typhimurium strains, with or without metabolic activation (Table 4). However, in previous studies, carvacrol, one of the components found in the oils with the highest antibacterial and antifungal activity, was demonstrated to be mutagenic on these strains [1]. In our experiments the non mutagenic activity of oregano essential oil could be probably due to the concomitant presence of different components which could be desmutagenic.

It was suggested that the essential oils are a promising food preservatives, because they show antimicrobial activity at low amounts almost always without any mutagenicity [4].

Data obtained in this study clearly showed the inhibitory activity of the essential oils against several pathogenic bacterial and fungal strains. On the other hand, these oils showed nor inhibitory activity against lactic acid bacteria neither mutagenic potential. Considered together, these findings can represent an important result, in the future view to use the essential oils as natural preservatives for food products, due to their positive effect relating to safety and shelf life. 
Table 3. Inhibition of fungal growth of essential oils: Melissa officinalis L. (balm), Ocimum basilicum L. (basil), Hyssopus officinalis L. (hyssop), Lavandula angustifolia Mill. (lavender), Origanum vulgare L. (oregano), Salvia officinalis L. (sage), Thymus vulgaris L. (thyme). Data are expressed in $\mathrm{cm}$ and do not include the diameter of paper disc. Results are shown as mean \pm standard deviation (SD) of the inhibition zone $(\mathrm{n}=3)$.

\begin{tabular}{|c|c|c|c|c|c|c|}
\hline $\begin{array}{l}\text { Essential oil } \\
\text { ( } \mu \mathrm{g} / \text { paper disc) }\end{array}$ & $\begin{array}{c}\text { Penicillium } \\
\text { simplicissimum }\end{array}$ & $\begin{array}{c}\text { Aureobasidium } \\
\text { pullulans }\end{array}$ & $\begin{array}{l}\text { Penicillium } \\
\text { citrinum }\end{array}$ & $\begin{array}{l}\text { Penicillium } \\
\text { expansum }\end{array}$ & $\begin{array}{c}\text { Debaryomyces } \\
\text { hansenii }\end{array}$ & $\begin{array}{c}\text { Penicillium } \\
\text { aurantiogriseum }\end{array}$ \\
\hline & $\mathrm{IZ}( \pm \mathrm{SD})$ & $\mathrm{IZ}( \pm \mathrm{SD})$ & $\mathrm{IZ}( \pm \mathrm{SD})$ & $\mathrm{IZ}( \pm \mathrm{SD})$ & $\mathrm{IZ}( \pm \mathrm{SD})$ & $\mathrm{IZ}( \pm \mathrm{SD})$ \\
\hline Balm $88 \mu \mathrm{g}$ & $0.60( \pm 0.00)$ & $0.27( \pm 0.06)$ & $0.57( \pm 0.06)$ & $\mathrm{NA}( \pm 0.00)$ & $\mathrm{NA}( \pm 0.00)$ & $0.30( \pm 0.00)$ \\
\hline Balm $177 \mu \mathrm{g}$ & $0.77( \pm 0.06)$ & $0.30( \pm 0.00)$ & $0.73( \pm 0.06)$ & $\mathrm{NA}( \pm 0.00)$ & $\mathrm{NA}( \pm 0.00)$ & $0.33( \pm 0.06)$ \\
\hline Balm $442 \mu \mathrm{g}$ & $1.43( \pm 0.06)$ & $0.53( \pm 0.12)$ & $1.43( \pm 0.31)$ & $\mathrm{NA}( \pm 0.00)$ & $\mathrm{NA}( \pm 0.00)$ & $1.00( \pm 0.10)$ \\
\hline Basil $96 \mu \mathrm{g}$ & $0.60( \pm 0.00)$ & $0.13( \pm 0.23)$ & $0.07( \pm 0.12)$ & $\mathrm{NA}( \pm 0.00)$ & $\mathrm{NA}( \pm 0.00)$ & $\mathrm{NA}( \pm 0.00)$ \\
\hline Basil $192 \mu \mathrm{g}$ & $0.77( \pm 0.06)$ & $0.37( \pm 0.06)$ & $0.20( \pm 0.17)$ & $\mathrm{NA}( \pm 0.00)$ & $\mathrm{NA}( \pm 0.00)$ & $\mathrm{NA}( \pm 0.00)$ \\
\hline Basil $480 \mu \mathrm{g}$ & $1.13( \pm 0.12)$ & $0.67( \pm 0.06)$ & $0.90( \pm 0.10)$ & $\mathrm{NA}( \pm 0.00)$ & $\mathrm{NA}( \pm 0.00)$ & $\mathrm{NA}( \pm 0.00)$ \\
\hline Hyssop 93 нg & $0.60( \pm 0.00)$ & $0.43( \pm 0.06)$ & $0.50( \pm 0.00)$ & $\mathrm{NA}( \pm 0.00)$ & $\mathrm{NA}( \pm 0.00)$ & $0.27( \pm 0.06)$ \\
\hline Hyssop $185 \mu \mathrm{g}$ & $0.77( \pm 0.6)$ & $0.53( \pm 0.06)$ & $0.60( \pm 0.00)$ & $\mathrm{NA}( \pm 0.00)$ & $\mathrm{NA}( \pm 0.00)$ & $0.30( \pm 0.00)$ \\
\hline Hyssop $463 \mu \mathrm{g}$ & $1.03( \pm 0.06)$ & $0.80( \pm 0.00)$ & $0.73( \pm 0.06)$ & $\mathrm{NA}( \pm 0.00)$ & $\mathrm{NA}( \pm 0.00)$ & $0.27( \pm 0.06)$ \\
\hline Lavender $87 \mu \mathrm{g}$ & $0.57( \pm 0.06)$ & $0.40( \pm 0.00)$ & $0.43( \pm 0.06)$ & $\mathrm{NA}( \pm 0.00)$ & $\mathrm{NA}( \pm 0.00)$ & $\mathrm{NA}( \pm 0.00)$ \\
\hline Lavender $177 \mu \mathrm{g}$ & $0.77( \pm 0.06)$ & $0.53( \pm 0.06)$ & $0.60( \pm 0.00)$ & $\mathrm{NA}( \pm 0.00)$ & $\mathrm{NA}( \pm 0.00)$ & $\mathrm{NA}( \pm 0.00)$ \\
\hline Lavender $443 \mu \mathrm{g}$ & $1.17( \pm 0.06)$ & $0.90( \pm 0.10)$ & $0.87( \pm 0.12) 1$ & $\mathrm{NA}( \pm 0.00)$ & $\mathrm{NA}( \pm 0.00)$ & $0.07( \pm 0.06)$ \\
\hline Oregano $93 \mu \mathrm{g}$ & $1.00( \pm 0.00)$ & $0.57( \pm 0.06)$ & $0.20( \pm 0.17)$ & $\mathrm{NA}( \pm 0.00)$ & $\mathrm{NA}( \pm 0.00)$ & $\mathrm{NA}( \pm 0.00)$ \\
\hline Oregano $187 \mu \mathrm{g}$ & $1.20( \pm 0.00)$ & $0.70( \pm 0.00)$ & $0.47( \pm 0.12)$ & $\mathrm{NA}( \pm 0.00)$ & $\mathrm{NA}( \pm 0.00)$ & $\mathrm{NA}( \pm 0.00)$ \\
\hline Oregano $467 \mu \mathrm{g}$ & $1.50( \pm 0.00)$ & $1.10( \pm 0.17)$ & $0.57( \pm 0.12)$ & $0.07( \pm 0.06)$ & $0.83( \pm 0.06)$ & $\mathrm{NA}( \pm 0.00)$ \\
\hline
\end{tabular}


Table 3. Cont.

\begin{tabular}{|c|c|c|c|c|c|c|}
\hline Sage $91 \mu \mathrm{g}$ & $0.50( \pm 0.00)$ & $\mathrm{NA}( \pm 0.00)$ & $0.50( \pm 0.00)$ & $\mathrm{NA}( \pm 0.00)$ & $\mathrm{NA}( \pm 0.00)$ & $\mathrm{NA}( \pm 0.00)$ \\
\hline Sage $183 \mu \mathrm{g}$ & $0.73( \pm 0.06)$ & $0.27( \pm 0.15)$ & $0.37( \pm 0.32)$ & $\mathrm{NA}( \pm 0.00)$ & $\mathrm{NA}( \pm 0.00)$ & $\mathrm{NA}( \pm 0.00)$ \\
\hline Sage $457 \mu \mathrm{g}$ & $1.07( \pm 0.12)$ & $0.23( \pm 0.06)$ & $0.90( \pm 0.10)$ & $\mathrm{NA}( \pm 0.00)$ & $0.23( \pm 0.06)$ & $\mathrm{NA}( \pm 0.00)$ \\
\hline Thyme $93 \mu \mathrm{g}$ & $0.77( \pm 0.06)$ & $0.93( \pm 0.06)$ & $0.57( \pm 0.06)$ & $\mathrm{NA}( \pm 0.00)$ & $\mathrm{NA}( \pm 0.00)$ & $\mathrm{NA}( \pm 0.00)$ \\
\hline Thyme $185 \mu \mathrm{g}$ & $0.87( \pm 0.06)$ & $1.07( \pm 0.12)$ & $0.80( \pm 0.10)$ & $\mathrm{NA}( \pm 0.00)$ & $\mathrm{NA}( \pm 0.00)$ & $\mathrm{NA}( \pm 0.00)$ \\
\hline Thyme $463 \mu \mathrm{g}$ & $1.60( \pm 0.40)$ & $1.47( \pm 0.06)$ & $1.17( \pm 0.15)$ & $\mathrm{NA}( \pm 0.00)$ & $\mathrm{NA}( \pm 0.00)$ & $\mathrm{NA}( \pm 0.00)$ \\
\hline DMSO & $\mathrm{NA}( \pm 0.00)$ & $\mathrm{NA}( \pm 0.00)$ & $\mathrm{NA}( \pm 0.00)$ & $\mathrm{NA}( \pm 0.00)$ & $\mathrm{NA}( \pm 0.00)$ & $\mathrm{NA}( \pm 0.00)$ \\
\hline
\end{tabular}

Table 4. Mutagenicity of essential oils: Melissa officinalis L. (balm), Ocimum basilicum L. (basil), Hyssopus officinalis L. (hyssop), Lavandula angustifolia Mill. (lavender), Origanum vulgare L. (oregano), Salvia officinalis L. (sage), Thymus vulgaris L. (thyme). Data are expressed as number of his + revertant colonies/plate (mean \pm standard deviation -SD-) in strains $S$. typhimurium TA98 and TA100 in presence $(+\mathrm{S} 9)$ and absence (-S9) of the metabolic activation.

\begin{tabular}{|c|c|c|c|c|}
\hline Essential oil & TA98 - S9 & TA100 - S9 & TA98+S9 & TA100 +S9 \\
\hline & CFU/Plate ( \pm SD) & CFU/Plate( \pm SD) & CFU/Plate ( \pm SD) & CFU/Plate ( \pm SD) \\
\hline Balm $88 \mu \mathrm{g}$ & $46.00( \pm 8.00)^{* * *}$ & $229.00( \pm 12.17) * * *$ & $53.00( \pm 6.56) * *$ & $393.00( \pm 14.73) *$ \\
\hline Balm $177 \mu \mathrm{g}$ & $47.67( \pm 5.86) * * *$ & $220.00( \pm 7.37) * * *$ & $50.33( \pm 5.03) * *$ & $352.67( \pm 11.24) * *$ \\
\hline Balm $442 \mu \mathrm{g}$ & $40.33( \pm 8.39) * * *$ & $175.00( \pm 29.82) * * *$ & $58.67( \pm 7.77) * *$ & $378.33( \pm 17.56) * *$ \\
\hline Basil $96 \mu \mathrm{g}$ & $52.67( \pm 4.62) * *$ & $209.00( \pm 61.88) * * *$ & $82.00( \pm 13.11) * * *$ & $81.33( \pm 18.04) * *$ \\
\hline Basil $192 \mu \mathrm{g}$ & $42.67( \pm 13.61) * * *$ & $217.00( \pm 27.87) * * *$ & $62.67( \pm 20.03) * * *$ & $83.00( \pm 8.19) * *$ \\
\hline Basil $480 \mu \mathrm{g}$ & $53.67( \pm 6.03) * * *$ & $156.67( \pm 25.32) * * *$ & $55.33( \pm 8.08) * * *$ & $110.67( \pm 21.01) * *$ \\
\hline Hyssop $93 \mu \mathrm{g}$ & $26.67( \pm 6.66) * * *$ & $256.33( \pm 22.59) * * *$ & $40.67( \pm 2.52) * * *$ & $357.00( \pm 24.02) * *$ \\
\hline Hyssop $185 \mu \mathrm{g}$ & $32.67( \pm 7.51) * * *$ & $226.00( \pm 26.91) * * *$ & $61.33( \pm 12.86) * * *$ & $293.33( \pm 91.09)$ * \\
\hline Hyssop $463 \mu \mathrm{g}$ & $35.00( \pm 5.00) * * *$ & $165.00( \pm 54.06) * * *$ & $56.33( \pm 4.73) * * *$ & $168.50( \pm 41.72) * *$ \\
\hline
\end{tabular}


Table 4. Cont.

\begin{tabular}{|c|c|c|c|c|}
\hline Lavender $87 \mu \mathrm{g}$ & $24.00( \pm 1.00) * * *$ & $107.33( \pm 6.81) * * *$ & $55.67( \pm 9.07) * * *$ & $90.00( \pm 27.84) * *$ \\
\hline Lavender $177 \mu \mathrm{g}$ & $41.33( \pm 7.77) * * *$ & $115.33( \pm 16.17) * * *$ & $39.67( \pm 8.96) * * *$ & $170.00( \pm 60.92) * *$ \\
\hline Lavender $443 \mu \mathrm{g}$ & $19.67( \pm 8.08) * * *$ & $103.67( \pm 13.87) * * *$ & $73.33( \pm 15.28) * * *$ & $255.00( \pm 5.00) *$ \\
\hline Oregano $93 \mu \mathrm{g}$ & $30.33( \pm 4.73) * * *$ & $170.00( \pm 43.59) * * *$ & $74.00( \pm 4.00) * * *$ & $277.00( \pm 12.12) * * *$ \\
\hline Oregano $187 \mu \mathrm{g}$ & $41.33( \pm 7.23) * * *$ & $174.00( \pm 48.99) * * *$ & $77.33( \pm 11.37) * * *$ & $342.00( \pm 92.37) *$ \\
\hline Oregano $467 \mu \mathrm{g}$ & $20.00( \pm 2.00) * * *$ & $121.00( \pm 8.08) * * *$ & $56.00( \pm 8.72) * * *$ & $308.33( \pm 64.22) *$ \\
\hline Sage $91 \mu \mathrm{g}$ & $54.00( \pm 6.00) * * *$ & $246.00( \pm 5.29) * * *$ & $63.33( \pm 14.43) * * *$ & $345.33( \pm 8.08) * *$ \\
\hline Sage $183 \mu \mathrm{g}$ & $45.33( \pm 2.52) * * *$ & $250.00( \pm 9.17) * * *$ & $45.00( \pm 7.55) * * *$ & $310.00( \pm 14.14) *$ \\
\hline Sage $457 \mu \mathrm{g}$ & $50.33( \pm 4.73) * * *$ & $203.00( \pm 20.82) * * *$ & $64.67( \pm 5.03) * * *$ & $310.33( \pm 26.84) * *$ \\
\hline Thyme 93 нg & $48.33( \pm 7.77) * * *$ & $212.00( \pm 13.86) * * *$ & $55.00( \pm 4.36) * * *$ & $292.33( \pm 10.69) * * *$ \\
\hline Thyme $185 \mu \mathrm{g}$ & $30.33( \pm 6.81) * * *$ & $193.00( \pm 12.22) * * *$ & $60.33( \pm 8.14)$ & $368.67( \pm 10.26) * *$ \\
\hline Thyme $463 \mu \mathrm{g}$ & $25.67( \pm 1.15) * * *$ & $214.00( \pm 22.50) * * *$ & $47.00( \pm 3.61)$ & $353.33( \pm 5.77) * *$ \\
\hline SR & $37.00( \pm 4.10)$ & $170.46( \pm 55.77)$ & $48.50( \pm 4.51)$ & $245.00( \pm 47.00)$ \\
\hline PC & $555.33( \pm 389.39)$ & $1108.58( \pm 356.67)$ & $159.87( \pm 55.31)$ & $562.50( \pm 74.40)$ \\
\hline
\end{tabular}

CFU: colony forming units; SR: spontaneous revertants used as negative control; PC: positive control, in presence of standard mutagen agents: for TA 98 (-S9), $o$-nitrofluorene (1 $\mu \mathrm{g} / \mathrm{plate})$; for TA100 (-S9), sodium azide (5 $\mu \mathrm{g} / \mathrm{plate})$; for TA98 (S9) and TA100 (S9), 2-aminoantracene (5 $\mu \mathrm{g} / \mathrm{plate)}$ ) * $\mathrm{p}<0.05$; $* * \mathrm{p}<0.01 ; * * * \mathrm{p}<0.001$ vs positive control. 


\section{Experimental}

\section{Essential oils}

Essential oils from the aerial parts of Ocimum basilicum L. (basil), Hyssopus officinalis L. (hyssop), Lavandula angustifolia Mill. (lavender), Melissa officinalis L. (balm), Origanum vulgare L. (oregano), Salvia officinalis L. (sage), Thymus vulgaris L. (thyme), were purchased from Azienda Chimica E Farmaceutica (A.C.E.F.) Spa (Fiorenzuola d'Arda, Italy). The density of the oils was furnished by A.C.E.F., as follows: Ocimum basilicum L. (0.954 g/mL), Hyssopus officinalis L. (0.926 g/mL), Lavandula angustifolia Mill. (0.893 g/mL lavender), Melissa officinalis L. (0.883 g/mL), Origanum vulgare L. (0.937 g/mL), Salvia officinalis L. (0.919 g/mL), Thymus vulgaris L. (0.927 g/mL).

\section{Chemical characterisation}

The oils were analysed by Gas Chromatography (GC) and Gas Chromatography-Mass Spectrometry (GC-MS). GC analyses were carried out using a Perkin-Elmer Sigma-115 gas chromatograph with a data handling system and a FID. Separation was achieved by using a fused-silica capillary column HP-5 MS, $30 \mathrm{~m}$ length, $0.25 \mathrm{~mm}$ internal diameter, $0.25 \mu \mathrm{m}$ film thickness. The operating conditions were as follows: injector and detector temperatures, $250{ }^{\circ} \mathrm{C}$ and $280{ }^{\circ} \mathrm{C}$, respectively; oven temperature programme: $5 \mathrm{~min}$ isothermal at $40{ }^{\circ} \mathrm{C}$, subsequently at $2{ }^{\circ} \mathrm{C} / \mathrm{min}$ up to $250{ }^{\circ} \mathrm{C}$ and finally raised to $270{ }^{\circ} \mathrm{C}$ at $10{ }^{\circ} \mathrm{C} \mathrm{min}-1$. Analysis was also run by using a fused silica $\mathrm{HP}$ Innowax polyethylene glycol capillary column $(50 \mathrm{~m} \times 0.20 \mathrm{~mm}$ i.d., $0.20 \mu \mathrm{m}$ film thickness). In both cases helium was used as the carrier gas $(1 \mathrm{~mL} / \mathrm{min})$. Diluted samples $(1 / 100 \mathrm{v} / \mathrm{v}$, in $n$ hexane $)$ of $1 \mu \mathrm{L}$ were injected manually at $250{ }^{\circ} \mathrm{C}$, and in the splitless mode.

GC-MS analysis was performed using an Agilent 6850 Ser. A apparatus, equipped with a fused silica HP-1 capillary column $(30 \mathrm{~m} \times 0.25 \mathrm{~mm}$ i.d.; film thickness $0.33 \mu \mathrm{m})$, linked on line with an Agilent Mass Selective Detector MSD 5973; ionization voltage 70 electron multiplier energy 2,000 V. Gas chromatographic conditions were as given above, transfer line was kept at $295{ }^{\circ} \mathrm{C}$. Most components were identified from their GC retention indices, with either those reported in literature [44-45] or with those of authentic compounds available in our laboratories, purchased from Sigma Aldrich, Co, Milan, Italy. The retention indices were determined in relation to a homologous series of $n$-alkanes $\left(\mathrm{C}_{8}-\mathrm{C}_{24}\right)$ under the same operating conditions. Further identification was made by comparison of their MS spectra on both columns with either mass spectra stored in NIST 02 and Wiley 275 libraries or with mass spectra from the literature $[44,46]$ and our homemade library. Component relative concentrations were calculated based on GC peaks without using correction factors. The percentage composition of the oils was computed by the normalization method from the GC peak areas, calculated as mean values of three injections from each oil, without using correction factors.

\section{Antibacterial activity}

The inhibition halos test on agar plate was employed to investigate the antibacterial activity of essential oils. The samples were tested against the following bacteria: non-pathogenic strains (Lactobacillus acidophilus DSM 20079; Lactobacillus casei DSM 9595; Lactobacillus bulgaricus 
DSM 20081; Lactobacillus sakei DSM 20494; Lactobacillus rhamnosus DSM 20711); pathogenic Gram positive strains Bacillus cereus (DSM 4313 and DSM 4384), Staphylococcus aureus DSM 25923 and Enterococcus faecalis DSM 2352; Gram negative strains, Escherichia coli DSM 8579 and Pseudomonas aeruginosa (ATCC 50071). All strains were purchased by Deutsche Sammlung von Mikroorganismen und Zellkulturen GmbH (DSMZ Germany). Each strain was incubated at $37{ }^{\circ} \mathrm{C}$ for $18 \mathrm{~h}$ into own specific growth medium: Lactic Acid Bacteria were grown in Man de Rogosa Sharpe (MRS) broth (Oxoid), E. coli, E. faecalis, S. aureus, P. aeruginosa and B. cereus in Nutrient Broth (Oxoid). The microbial suspensions $\left(1 \times 10^{8}\right.$ Colony Forming Units-CFU-/mL) were uniformly spread onto the specific solid media plates $\left(\varnothing=90 \mathrm{~mm}\right.$ dishes). Sterile Whatman $\mathrm{n}^{\circ} 1$ paper filter discs $(\varnothing=5 \mathrm{~mm})$ were individually placed on the inoculated plates and impregnated with different amounts of essential oils, previously diluted 1:10 (v/v) in dimethylsulfoxide (DMSO) (final amount ranging from 88 to $490 \mu \mathrm{g} /$ paper disc). The exact amounts of each oils are reported in Table 2 . After $30 \mathrm{~min}$ under sterile conditions at room temperature, plates were incubated at $37^{\circ} \mathrm{C}$ for $24-48 \mathrm{~h}$, depending on the strain. The diameter of the clear zone shown on plates was accurately measured and the antibacterial activity expressed in $\mathrm{cm}$ (not including disc diameter of $0.5 \mathrm{~cm}$ ). Sterile deionised water and DMSO (10 $\mu \mathrm{L} /$ paper disc) were used as negative control. Gentamycin (8 $\mu \mathrm{g} /$ paper disc), chloramphenicol (66 $\mu \mathrm{g}$ /paper disc) and tetracycline (7 $\mu \mathrm{g} /$ paper disc), in physiological solution, served as positive controls. Samples were tested in triplicate and results expressed as mean \pm standard deviation.

\section{Antifungal activity}

The inhibition halos test on agar plate was employed to investigate the antifungal activity of the essential oils. Six fungal strains of agro-food interest (Penicillium citrinum DSM 1997, Penicillium simplicissimum DSM 1097, Aureobasidium pullulans DSM 62074, Penicillium expansum DSM 1994, Penicillium aurantiogriseum DSM 2429, Debaryomyces hansenii DSM 70238), were used. All strains were purchased by DSMZ. Sterile Whatman $n^{\circ} 1$ paper filter discs $(\varnothing=5 \mathrm{~mm})$ were individually placed on the inoculated plates ( $\varnothing=90 \mathrm{~mm}$ dishes) and impregnated with different amounts of essential oils previously diluted 1:10 (v/v) in DMSO (final amount ranging from 88 to $490 \mu \mathrm{g} /$ paper disc), were used. The exact amounts of each oils are reported in Table 3. A cell suspension of fungi was prepared in sterile distilled water, adjusted to contain approximately $10^{6} \mathrm{CFU} / \mathrm{mL}$, and plated onto Potato Dextrose Agar (PDA) (Oxoid). After 20 min under sterile conditions at room temperature, plates were incubated at $28{ }^{\circ} \mathrm{C}$ for $48-72 \mathrm{~h}$. When the mycelium of fungi reached the edges of the control plate (negative control without the samples added extracts), the diameter of the clear zone shown on plates was accurately measured and the antifungal activity expressed in $\mathrm{cm}$ (not including disc diameter of $0.5 \mathrm{~cm}$ ). DMSO was used as negative control $(10 \mu \mathrm{L}$ /paper disc). Samples were tested in triplicate and the results are expressed as mean \pm standard deviation.

\section{Mutagenic activity}

For mutagenicity assay, the bacteria reverse mutation assay (Ames test) plate incorporation method, was performed [47] by using the Salmonella typhimurium histidine-requiring strains TA98 and TA100, 
purchased from Molecular Toxicology Inc. (Moltox ${ }^{\mathrm{TM}}$, Annapolis, MD, USA). Strains were aerobically allowed to grow overnight at $37^{\circ} \mathrm{C}$ in Nutrient Broth $\mathrm{n}^{\circ} 2$ (Oxoid) supplemented with ampicillin. With the aim to define the amount of the essential oils that did not give cytotoxic effect, a preliminary test was performed by up to $900 \mu \mathrm{g}$ /plate of each oil. The toxicity was evaluated by the complete absence of background lawn [48]. The test was carried out by addition to $1.8 \mathrm{~mL}$ of molten agar of the following: $0.1 \mathrm{~mL}$ of the overnight bacterial culture, different amounts of essential oils previously diluted 1:10 (v/v) in DMSO (final amount ranging from 88 to $490 \mu \mathrm{g}$ ), $0.5 \mathrm{~mL}$ phosphate buffer (0.1 M, pH 7.4) or $0.5 \mathrm{~mL}$ of the metabolic activation S9 mixture. The exact amounts of each oil are reported in Table 4. The mixture was immediately plated onto minimal medium agar plates ( $\varnothing=90 \mathrm{~mm}$ dishes) (previously added with $0.2 \mathrm{~mL}$ of sterile $0.5 \mathrm{mM}$ Bio-His) and incubated at $37{ }^{\circ} \mathrm{C}$ for $48 \mathrm{~h}$ in the dark. The rat liver post-mitocondrial supernatant fraction (S9) $[47,48]$ induced by a polychlorinated biphenyl mixture, Aroclor 1254 (0.7 nmol cytochrome P450/mg protein), in male Sprague-Dawley rats, used for the indirect test, was purchased from Moltox ${ }^{\mathrm{TM}}$ (Annapolis, MD, USA). As positive controls, the direct acting mutagens $o$-nitrofluorene $(1 \mu \mathrm{g} / \mathrm{plate})$ was used for strain TA98 and sodium azide (5 $\mu \mathrm{g} / \mathrm{plate})$ was used for strain TA100; the indirect acting mutagen 2-aminoantracene $(5 \mu \mathrm{g} /$ plate) was used for both TA98 and TA100 stains. DMSO (10 $\mu \mathrm{L} /$ plate) was used as negative control. Samples were tested in triplicate and the results are expressed as mean \pm standard deviation.

\section{Statistical analysis}

Data were ordered in homogeneous sets, and the Student's $t$ test of independence was applied [49].

\section{Conclusion}

Data obtained showed the inhibitory activity of the essential oils assayed against pathogenic bacterial and fungal strains. On the other hand, these oils showed no inhibitory activity against lactic acid bacteria. These findings, considered together, can represent an important result, in the future view to use the essential oils as natural preservatives for food products, due to their positive effect on their safety and shelf life.

\section{References}

1. Ipek, E.; Zeytinoglu, H.; Okay, S.; Tuylu, B.A.; Kurkcuoglu, M.; Baser, K.H.C. Genotoxicity and antigenotoxicity of Origanum oil and carvacrol evaluated by Ames Salmonella/microsomal test. Food Chem. 2005, 93, 551-556.

2. Evandri, M.G.; Battinelli, L.; Daniele, C.; Mastrangelo, S.; Bolle, P.; Mazzanti, G. The antimutagenic activity of Lavandula angustifolia (lavender) essential oil in the bacterial reverse mutation assay. Food Chem. Toxicol. 2005, 43, 1381-1387.

3. Bozin, B.; Mimica-Dukic, N.; Simin, N.; Anackov, G. Characterization of the volatile composition of essential oils of some Lamiaceae spices and the antimicrobial and antioxidant activities of the entire oils. J. Agric. Food Chem. 2006, 54, 1822-1828.

4. Tsigarida, E.; Skandamis, P.; Nychas, G.J.E. Behaviour of Lysteria monocytogenes andautochthonous flora on meat stored under aerobic, vacuum and modified atmosphere 
packaging conditions with or without the presence of oregano essential oil at $5{ }^{\circ} \mathrm{C} . \mathrm{J}$. Appl. Microbiol. 2000, 89, 901-909.

5. Hammer, K.A.; Carson, C.F.; Riley, T.V. Antimicrobial activity of essential oils and other plant extracts. J. Appl. Microbiol. 1999, 86, 985-990.

6. Aureli, P.; Costantini, A.; Zolea, S. Antimicrobial activity of some plant essential oils against Listeria monocytogenes. J. Food Prot. 1992, 55, 334-348.

7. Cosentino, S.; Tuberoso, C.I.G.; Pisano, B.; Satta, M.; Mascia, V.; Arzedi, E.; Palmas, F. In vitro antimicrobial activity and chemical composition of Sardinian Thymus essential oils. Lett. Appl. Microbiol. 1999, 29, 130-135.

8. Dorman, H.J.D.; Deans, S.G. Antimicrobial agents from plants: Antibacterial activity of plant volatile oils. J. Appl. Microbiol. 2000, 88, 308-316.

9. Di Pasqua, R.; De Feo, V.; Villani, F.; Mauriello, G. In vitro antimicrobial activity of essential oils from Mediterranean Apiaceae, Verbenaceae and Lamiaceae against foodborne pathogens and spoilage bacteria. Ann. Microbiol. 2005, 19, 226-230.

10. Chericoni, S.; Prieto, J.M.; Iacopini, P.; Ciono, P.; Morelli, I. In vitro activity of the essential oil of Cinnamomum zeylancium and eugenol in peroxynitrite-induced oxidative processes. J. Agric. Food Chem. 2005, 53, 4762-4765.

11. Chao, L.K.; Hua, K.; Hsu, H.; Cheng, S.; Liu, J.; Chang, S. Study on the antiinflammatory activity of essential oil from leaves of Cinnamomum osmophloeum. J. Agric. Food Chem. 2005, 53, 7274-7278.

12. Singh, G.; Marimuthu, P.; De Heluani, C.S.; Catalan, C.A.N. Antioxidant and biocidal activities of Carum nigrum (Clonal) essential oil, oleoresin, and their selected components. J. Agric. Food Chem. 2006, 54, 174-181.

13. Vizoso A.; Ramos A.; Villaescusa A.; Decalo M.; Betancourt J. Estudio genotoxico in vitro e in vivo en tinturas de Melissa officinalis L (Toronjíl) y Menta piperita L (Toronjíl de Menta). Rev. Cub. Planta Med. 1997, 2, 6-11.

14. Vuković-Gačić, B.; Nikčević, S.; Berić-Bjedov, T.; Knežević-Vukčević, J.; Simić, D. Antimutagenic effect of essential oil of sage (Salvia officinalis L.) and its monoterpenes against UV-induced mutations in Escherichia coli and Saccharomyces cerevisiae. Food Chem. Toxicol. 2006, 44, 1730-1738.

15. Senatore, F.; De Fusco, R.; De Feo, V. Essential oils from Salvia spp. (Lamiaceae) I Chemical composition and antimicrobial screening of essential oils from Salvia glutinosa L. growing wild in Southern Italy. J. Essent. Oil Res. 1997, 9, 151-157.

16. De Feo, V.; Bruno, M.; Napolitano, F.; Tahiri, B.; Senatore, F. Chemical composition and antibacterial activity of essential oils from Thymus spinulosus Ten. (Lamiaceae). J. Agric. Food Chem. 2003, 51, 3849-3853.

17. Arminante, F.; De Falco, E.; De Feo, V.; De Martino, L.; Mancini, E.; Quaranta, E. Allelopathic activity of essential oils from Mediterranean Lamiaceae. Acta Hort. 2006, 723, 347-356.

18. Stefanini, I.; Piccaglia, R.; Marotti, M.; Biavati, B. Characterization and biological activity of essential oils from fourteen Lamiaceae species. Acta Hort. 2006, 723, 221-226.

19. Hasegawa, Y.; Tajima, K.; Toi, N.; Sugimura, Y. Characteristic components found in the essential oil of Ocimum basilicum L. Flavour Frag. J. 1997, 12, 195-200. 
20. Basta, A.; Tzakou, O.; Couladis, M. Composition of the leaves essential oil of Melissa officinalis s 1 from Greece. Flavour Frag. J. 2005, 20, 642-644.

21. Lee, S.J.; Umano, K.; Shibamoto, T.; Lee, K.G. Identification of volatile components in basil (Ocimum basilicum L.) and thyme leaves (Thymus vulgaris L.) and their antioxidant properties. Food Chem. 2005, 91, 131-137.

22. Chalchat, J.C.; Öezcan, M.M. Comparative essential oil composition of flowers, leaves and stems of basil (Ocimum basilicum L.) used as herb. Food Chem. 2008, 110, 501-503.

23. Mazzanti, G.; Battinelli, L.; Salvatore, G. Antimicrobial properties of the linalol-rich essential oil of Hyssopus officinalis L. var decumbens (Lamiaceae). Flavour Frag. J. 1998, 13, 289-294.

24. Morgan, T.J.; Morden, W.E.; Al-Muhareb, E.; Herod, A.A.; Kandiyoti, R. Essential oils investigated by size exclusion chromatography and gas chromatography-mass spectrometry. Energ. Fuel. 2006, 20, 734-737.

25. García, M.A.; Sanz, J. Analysis of Origanum vulgare volatiles by direct thermal desorption coupled to gas chromatography-mass spectrometry. J. Chromatogr. A 2001, 918, 189-194.

26. Veres, K.; Varga, E.; Dobos, A.; Hajdú, Z.S.; Máthé, I.; Németh, E.; Szabó, K. Investigation of the composition and stability of the essential oils of Origanum vulgare ssp. vulgare L. and $O$. vulgare ssp. hirtum (Link) Ietswaart. Chromatographia 2003, 57, 95-98.

27. Hudaib, M.; Speroni, E.; Di Pietra, A.M.; Cavrini, V. GC/MS evaluation of thyme (Thymus vulgaris L.) oil composition and variations during the vegetative cycle. J. Pharm. Biomed. Anal. 2002, 29, 691-700.

28. Angelini, L.G.; Carpanese, G.; Cioni, P.L.; Morelli, I.; Macchia, M.; Flamini, G. Essential oils from Mediterranean Lamiaceae as weed germination inhibitors. J. Agric. Food Chem. 2003, 51, $6158-6164$.

29. Veličković, A.S.; Ristić, M.S.; Veličković, D.T.; Ilić, S.N.; Mitić, N.D. The possibilities of the application of some species of sage (Salvia L.) as auxiliaries in the treatment of some diseases. $J$. Serb. Chem. Soc. 2003, 68, 435-445.

30. Lima, C.F.; Carvalho, F.; Fernandes, E.; Bastos, M.L.; Santos-Gomes, P.C.; Fernandes-Ferreira, M.; Pereira-Wilson, C. Evaluation of toxic/protective effects of the essential oil of Salvia officinalis on freshly isolated rat hepatocytes. Toxicol. In Vitro 2004, 18, 457-465.

31. Valero, M.; Salmerón, M.C. Antibacterial activity of 11 essential oils against Bacillus cereus in tyndallized carrot broth. Int. J. Food Microbiol. 2003, 85, 73-81.

32. Valero, M.; Giner, M.J. Effects of antimicrobial components of essential oils on growth of Bacillus cereus INRA L2104 in and the sensory qualities of carrot broth. Int. J. Food Microbiol. 2006, 106, 90-94.

33. Verluyten, J.; Leroy, F.; De Vuyst, L. Effects of different spices used in production of fermented sausages on growth of and curvacin a production by Lactobacillus curvatus LTH 1174. Appl. Environ. Microbiol. 2004, 70, 4807-4813.

34. Andrews, J.H.; Spear, R.N.; Nordheim, E.V. Population biology of Aureobasidium pullulans on apple leaf surfaces. Can. J. Microbiol. 2002, 48, 500-513.

35. Maggi, R.G.; Govind, N.S. Regulated expression of green fluorescent protein in Debaryomyces hansenii. J. Ind. Microbiol. Biotechnol. 2004, 31, 301-310. 
36. Xu, B.J.; Jia, X.Q.; Gu, L.J.; Sung, C.K. Review on the qualitative and quantitative analysis of the mycotoxin citrinin. Food Control 2006, 17, 271-285.

37. Mishra, A.K.; Dwivedi, S.K.; Kishore, N. Antifungal activity of some essential oils. Nat. Acad. Sci. Lett. 1989, 12, 335-336.

38. Pauli, A.; Knobloch, K. Inhibitory effects of essential oil components on growth of foodcontamining fungi. Z. Lebensm. Unters. Forsch. 1987, 185, 10-13.

39. Letessier, M.P.; Svoboda, K.P.; Walters, D.R. Antifungal activity of the essential oil of Hyssop (Hyssopus officinalis). J. Phytopatol. 2001, 149, 673-678.

40. Pina-Vaz, C.; Gonçalves-Rodrigues, A.; Pinto, E.; Costa-De-Oliveira, S.; Tavares, C.; Salgueiro, L.; Cavaleiro, C.; Goncalves, M.J.; Martinezde-Oliveira, J. Antifungal activity of Thymus oils and their major compounds. J. Eur. Acad. Dermatol. Venereol. 2004, 18, 73-78.

41. Pinto, E.; Ribeiro, Salgueiro, L.; Cavaleiro, C.; Palmeira, A.; Gonçalves, M.J. In vitro susceptibility of some species of yeasts and filamentous fungi to essential oils of Salvia officinalis. Ind. Crop. Prod. 2007, 26, 135-141.

42. Adam, K.; Sivropoulou, A.; Kokkini, S.; Lanaras, T.; Arsenakis, M. Antifungal activities of Origanum vulgare subsp. hirtum, Mentha spicata, Lavandula angustifolia, and Salvia fruticosa essential oils against human pathogenic fungi. J. Agric. Food Chem. 1998, 46, 1739-1745.

43. Smid, E.J.; Dewitte, Y.; Gorris, L.G.M. Secondary plant metabolites as control agents of postharvest Penicillium rot on tulip bulbs. Postharvest Biol. Technol. 1995, 6, 303-312.

44. Jennings, W.; Shibamoto T. Qualitative Analysis of Flavour and Fragrance Volatiles by Glass Capillary Gas Chromatography; Academic Press: New York, NY, USA, 1980.

45. Davies, N.W. Gas chromatographic retention indices of monoterpenes and sesquiterpenes on methyl silicone and Carbowax 20M phases. J. Chromatogr. 1990, 503, 1-24.

46. Adams R.P. Identification of Essential Oil Components by Gas Chromatography/Mass Spectrometry; Allured Publishing: Carol Stream, IL, USA, 2001.

47. Maron, D.M.; Ames, B.N. Revised methods for the Salmonella mutagenicity test. Mut. Res. 1983, 113, 173-215.

48. Mortelmans, K.; Zeiger, E. The Ames Salmonella/microsome mutagenicity assay. Mut. Res. 2000, 455, 29-60.

49. Sokal, R.R.; Rohlf, F.J. Biometry, 2nd Ed.; WH Freeman and Company: New York, NY, USA, 1981.

Sample Availability: Samples of the essential oils are available from the authors.

(C) 2009 by the authors; licensee Molecular Diversity Preservation International, Basel, Switzerland. This article is an open-access article distributed under the terms and conditions of the Creative Commons Attribution license (http://creativecommons.org/licenses/by/3.0/). 